PROCEEDINGS OF THE

AMERICAN MATHEMATICAL SOCIETY

Volume 133, Number 11, Pages 3153-3162

S 0002-9939(05)07956-6

Article electronically published on May 2, 2005

\title{
CODIMENSION THEOREMS FOR COMPLETE TORIC VARIETIES
}

\author{
DAVID COX AND ALICIA DICKENSTEIN
}

(Communicated by Michael Stillman)

\begin{abstract}
Let $X$ be a complete toric variety with homogeneous coordinate ring $S$. In this article, we compute upper and lower bounds for the codimension in the critical degree of ideals of $S$ generated by $\operatorname{dim}(X)+1$ homogeneous polynomials that do not vanish simultaneously on $X$.
\end{abstract}

\section{INTRODUCTION}

In this paper, $X$ will denote a complete toric variety of dimension $n$. We will work over $\mathbb{C}$, so that the torus of $X$ is $\left(\mathbb{C}^{*}\right)^{n}$. The dual lattices will be denoted by $M$ and $N$ as usual, and the minimal edge generators of the fan of $X$ will be denoted by $n_{1}, \ldots, n_{r} \in N$. Corresponding to each $n_{i}$ we have the irreducible torus-invariant divisor $D_{i}$ and the variable $x_{i}$ in the homogeneous coordinate ring $S=\mathbb{C}\left[x_{1}, \ldots, x_{r}\right]$, which is graded by the Chow group $A_{n-1}(X)$.

Consider a torus-invariant Cartier divisor $D=\sum_{i} a_{i} D_{i}$ such that $\mathcal{O}_{X}(D)$ is generated by global sections. In Section 1, we recall a vanishing theorem of Batyrev and Borisov [2] that describes $H^{i}\left(X, \mathcal{O}_{X}(-D)\right)$ in terms of the polytope

$$
\Delta_{D}=\left\{m \in M_{\mathbb{R}} \mid\left\langle m, n_{i}\right\rangle \geq-a_{i}\right\} .
$$

By Serre duality, we get a description of

$$
H^{i}\left(X, \mathcal{O}_{X}(D+K)\right),
$$

where $K$ is the canonical class.

In Section 2 we consider homogeneous polynomials $f_{0}, \ldots, f_{n} \in S$ of degrees $\alpha_{0}, \ldots, \alpha_{n} \in A_{n-1}(X)$ which satisfy the following two properties: first, the $f_{i}$ do not vanish simultaneously on $X$, and second, each $\alpha_{i}$ lies in $\operatorname{Pic}(X)$ and the corresponding line bundle $\mathcal{O}_{X}\left(\alpha_{i}\right)$ is generated by global sections. By abuse of terminology, we will say that $\alpha_{i}$ is globally generated in this situation.

Received by the editors November 10, 2003 and, in revised form, June 14, 2004.

2000 Mathematics Subject Classification. Primary 14M25.

Key words and phrases. Toric variety.

The first author thanks the Mathematics Department of the University of Buenos Aires for their hospitality during his visits there in 2001 and 2003.

The second author was supported by ANPCYT 03-06568, UBACYT X-052 and Conicet, Argentina.

(C)2005 American Mathematical Society Reverts to public domain 28 years from publication 
Following [4, we define the critical degree to be

$$
\rho=\sum_{i=0}^{n} \alpha_{i}-\beta_{0}
$$

where $\beta_{0}=\sum_{j=1}^{r} \operatorname{deg}\left(x_{j}\right)$, and we let

$$
I=\left\langle f_{0}, \ldots, f_{n}\right\rangle \subset S
$$

be the homogeneous ideal generated by the $f_{i}$. Then the Codimension Question asks what is the dimension of $(S / I)_{\rho}$, i.e., what is the codimension of $I_{\rho}$ in $S_{\rho}$ ?

The main result of Section 2 computes upper and lower bounds for this codimension, when the associated family of polytopes is essential. In particular, it is always nonzero. The assumption of having an essential family is natural since it is necessary for the existence of a nontrivial sparse resultant whose vanishing is equivalent to the condition that the $f_{i}$ do not have common zeros on $X$ (see [11). We also give further geometric conditions on the polytopes under which the codimension at the critical degree attains the upper bound that we present.

When the divisors involved are big and nef, our results imply that the codimension is 1 . The first toric codimension-one theorem, proved in [7] using ideas from [1], assumed that the $\alpha_{i}$ were all equal to the class of a single ample divisor. This was extended in [5] to the case when the $\alpha_{i}$ were positive integer multiples of a single ample class. In the general case when the $\alpha_{i}$ are all ample, a codimension-one theorem was proved in [4 provided that $X$ was simplicial. All of these results now follow from part (1) of Corollary 2.5.

The codimension in the critical degree is important because of its relation to the theory of toric residues $3,4,4,5,7,8$. Toric residues are rational functions of the coefficients of the given polynomials $f_{0}, \ldots, f_{n}$; moreover, they are rational hypergeometric functions determined by the lattice points of the associated family of polytopes, with poles at the resultant locus [6]. As described in [7], a homogeneous polynomial $H$ of critical degree gives rise to a rational $n$-form on $X$. Since the $f_{i}$ do not vanish simultaneously on $X$, this $n$-form represents an element of $H^{n}\left(X, \widehat{\Omega}_{X}^{n}\right) \simeq H^{n}\left(X, \mathcal{O}_{X}\left(-\beta_{0}\right)\right)$. The toric residue of $H$ is defined to be the trace of this cohomology class. If the codimension is one and we have an explicit element $J$ of critical degree with known residue, then the computation of the toric residue of $H$ is reduced to writing $H=c J$ modulo $I$. When all degrees are equal and ample, a choice of $J$ is the toric Jacobian [7] associated to $f_{0}, \ldots, f_{n}$. In case all degrees are ample, explicit elements with residue equal to \pm 1 are known [4, 8, but it is still an open problem to find such elements in the big and nef but not ample case.

\section{The VAnishing THEOREM}

Now let $D$ be a torus-invariant Cartier divisor on a complete toric variety $X$. We define the polytope $\Delta_{D}$ as in the introduction and we use $\operatorname{int}\left(\Delta_{D}\right)$ to denote the relative interior of $\Delta_{D}$. Here is the vanishing theorem from [2, Thm. 2.5].

Theorem 1.1. Let $D$ be a torus-invariant Cartier divisor on a complete toric variety $X$ and let $\Delta_{D}$ be the polytope defined above. If $\mathcal{O}_{X}(D)$ is generated by global sections, then:

(1) $H^{i}\left(X, \mathcal{O}_{X}(-D)\right)=0$ for $i \neq \operatorname{dim}\left(\Delta_{D}\right)$. 
(2) There is an isomorphism

$$
H^{\operatorname{dim}\left(\Delta_{D}\right)}\left(X, \mathcal{O}_{X}(-D)\right) \simeq \bigoplus_{m \in M \cap \operatorname{int}\left(\Delta_{D}\right)} \mathbb{C} \cdot \chi^{-m}
$$

which is equivariant with respect to the natural $\left(\mathbb{C}^{*}\right)^{n}$ action on each side.

Remark 1.2. We should note that for a complete toric variety $X$, the sheaf $\mathcal{O}_{X}(D)$ is generated by its global sections if and only if $D$ is a nef divisor (this observation appears in [9] and [10]). Also, in the terminology of [9], such a divisor $D$ is called $\ell$-semiample, where $\ell=\operatorname{dim}\left(\Delta_{D}\right)$.

Using Serre Duality, we get the following corollary of Theorem 1.1. As usual, $K=K_{X}$ denotes the canonical divisor of $X$.

Corollary 1.3. Under the same hypotheses as Theorem 1.1, we have:

(1) $H^{i}\left(X, \mathcal{O}_{X}(D+K)\right)=0$ for $i \neq n-\operatorname{dim}\left(\Delta_{D}\right)$.

(2) There is an isomorphism

$$
H^{n-\operatorname{dim}\left(\Delta_{D}\right)}\left(X, \mathcal{O}_{X}(D+K)\right) \simeq \bigoplus_{m \in M \cap \operatorname{int}\left(\Delta_{D}\right)} \mathbb{C} \cdot \chi^{m}
$$

which is equivariant with respect to the natural $\left(\mathbb{C}^{*}\right)^{n}$ action on each side.

Proof. We know that $K$ is given by the Weil divisor $-\sum_{j=1}^{r} D_{i}$. By Serre Duality,

$$
H^{i}\left(X, \mathcal{O}_{X}(D) \otimes_{\mathcal{O}_{X}} \mathcal{O}_{X}(K)\right) \simeq H^{n-i}\left(X, \mathcal{O}_{X}(-D)\right)^{*} .
$$

However, given any Weil divisors $E$ and $F$ on $X$, the natural map

$$
\mathcal{O}_{X}(E) \otimes_{\mathcal{O}_{X}} \mathcal{O}(F) \rightarrow \mathcal{O}_{X}(E+F)
$$

is easily seen to be an isomorphism when $E$ or $F$ is Cartier. Since $D$ is Cartier by assumption, the above duality may be written

$$
H^{i}\left(X, \mathcal{O}_{X}(D+K)\right) \simeq H^{n-i}\left(X, \mathcal{O}_{X}(-D)\right)^{*} .
$$

By functoriality, this is compatible with the $\left(\mathbb{C}^{*}\right)^{n}$ action on everything. From here, the corollary follows immediately from Theorem 1.1

Remark 1.4. When $D$ is big and nef, we have $\operatorname{dim}\left(\Delta_{D}\right)=n$. Then Corollary 1.3 implies that

$$
H^{i}\left(X, \mathcal{O}_{X}(D+K)\right)=0, \quad i>0 .
$$

This is the Kawamata-Viehweg vanishing theorem from [10].

\section{Codimension in the CRitical Degree}

As in the introduction, fix $\alpha_{0}, \ldots, \alpha_{n} \in \operatorname{Pic}(X) \subset A_{n-1}(X)$ such that each $\alpha_{i}$ is globally generated. This implies that each $\alpha_{i}$ determines a lattice polytope $\Delta_{i}$ which is well-defined up to translation by an element of $M$.

Given polynomials $f_{i} \in S_{\alpha_{i}}$ (so that $\operatorname{deg}\left(f_{i}\right)=\alpha_{i}$ ), we obtain the homogeneous ideal $I=\left\langle f_{0}, \ldots, f_{n}\right\rangle \subset S$. We want to study the codimension of $I_{\rho}$ in $S_{\rho}$, where $\rho=\sum_{i=0}^{n} \alpha_{i}-\beta_{0}$ is the critical degree (0.1).

Before stating our main result we need a definition. 
Definition 2.1. Let $\Delta_{i}, i \in I$, be polytopes in $\mathbb{R}^{n}$. Given $J \subset I$, set

$$
\Delta_{J}=\sum_{j \in J} \Delta_{j}
$$

The family $\left\{\Delta_{i}, i \in I\right\}$ is called essential if for every $J \subset I$ with $|J| \leq n$, we have

$$
\operatorname{dim}\left(\Delta_{J}\right) \geq|J| .
$$

We will also use the standard notation

$$
l^{*}(\Delta)=\#(M \cap \operatorname{int}(\Delta))
$$

to denote the number of lattice points of a polytope $\Delta$ which lie in the relative interior of $\Delta$.

Here is our codimension theorem.

Theorem 2.2. Suppose that $X$ is a complete toric variety of dimension $n$. Let $\alpha_{i} \in \operatorname{Pic}(X), 0 \leq i \leq n$, be globally generated and assume that the corresponding family of polytopes $\left\{\Delta_{i}, 0 \leq i \leq n\right\}$ is essential. If $f_{i} \in S_{\alpha_{i}}, 0 \leq i \leq n$, do not vanish simultaneously on $X$, then:

(1) The codimension of $I_{\rho}$ in $S_{\rho}$ satisfies the inequalities

$$
1+\sum_{\operatorname{dim}\left(\Delta_{\ell}\right)=1} l^{*}\left(\Delta_{\ell}\right) \leq \operatorname{dim}\left((S / I)_{\rho}\right) \leq 1+\sum_{k=1}^{n-1} \sum_{\operatorname{dim}\left(\Delta_{J}\right)=|J|=k} l^{*}\left(\Delta_{J}\right),
$$

where in the right-most sum we always assume that $J \subset\{0, \ldots, n\}$.

(2) The codimension of $I_{\rho}$ in $S_{\rho}$ is given by

$$
\operatorname{dim}\left((S / I)_{\rho}\right)=1+\sum_{k=1}^{n-1} \sum_{\operatorname{dim}\left(\Delta_{J}\right)=|J|=k} l^{*}\left(\Delta_{J}\right)
$$

if one of the following is satisfied for every $J$ with $1 \leq|J| \leq n-2$ :

(a) $\operatorname{dim}\left(\Delta_{J}\right) \neq|J|+1$;

(b) $\operatorname{dim}\left(\Delta_{J}\right)=|J|+1$ but $\Delta_{J}$ has no interior lattice points;

(c) $\operatorname{dim}\left(\Delta_{J}\right)=|J|+1$ but $\operatorname{dim}\left(\Delta_{J \cup I}\right)>|J|+|I|$ for nonempty subsets $I \subset\{0, \ldots, n\}$ such that $I \cap J=\emptyset$ and $|J|+|I|<n$.

Proof. Since $f_{i}$ is a global section of $\mathcal{O}_{X}\left(\alpha_{i}\right)$, we get a Koszul complex

$$
0 \rightarrow \mathcal{O}_{X}\left(-\sum_{i} \alpha_{i}\right) \rightarrow \cdots \rightarrow \bigoplus_{i} \mathcal{O}_{X}\left(-\alpha_{i}\right) \rightarrow \mathcal{O}_{X} \rightarrow 0
$$

which is exact since each $\mathcal{O}_{X}\left(\alpha_{i}\right)$ is locally free and the $f_{i}$ have no common zeros.

By hypothesis, each sheaf in the Koszul complex is locally free. Since the sheaf $\operatorname{Tor}_{i}^{\mathcal{O}} x(\mathcal{E}, \mathcal{F})$ vanishes whenever $i>0$ and $\mathcal{E}$ or $\mathcal{F}$ is locally free, it follows that the Koszul sequence remains exact after tensoring with $\mathcal{O}(\rho)=\mathcal{O}\left(\alpha_{0}+\cdots+\alpha_{n}-\beta_{0}\right)$ (which need not be locally free). This gives the exact sequence

$$
0 \rightarrow \mathcal{O}_{X}\left(-\sum_{i} \alpha_{i}\right) \otimes_{\mathcal{O}_{X}} \mathcal{O}_{X}(\rho) \rightarrow \cdots \rightarrow \bigoplus_{i} \mathcal{O}_{X}\left(-\alpha_{i}\right) \otimes_{\mathcal{O}_{X}} \mathcal{O}_{X}(\rho) \rightarrow \mathcal{O}_{X}(\rho) \rightarrow 0 .
$$

Since the $\alpha_{i}$ all come from Cartier divisors, the reasoning used in the proof of Corollary 1.3 implies that we can write this exact sequence as

$$
0 \rightarrow \mathcal{O}_{X}\left(-\beta_{0}\right) \rightarrow \bigoplus_{i} \mathcal{O}_{X}\left(\alpha_{i}-\beta_{0}\right) \rightarrow \cdots \rightarrow \mathcal{O}_{X}\left(\alpha_{0}+\cdots+\alpha_{n}-\beta_{0}\right) \rightarrow 0
$$




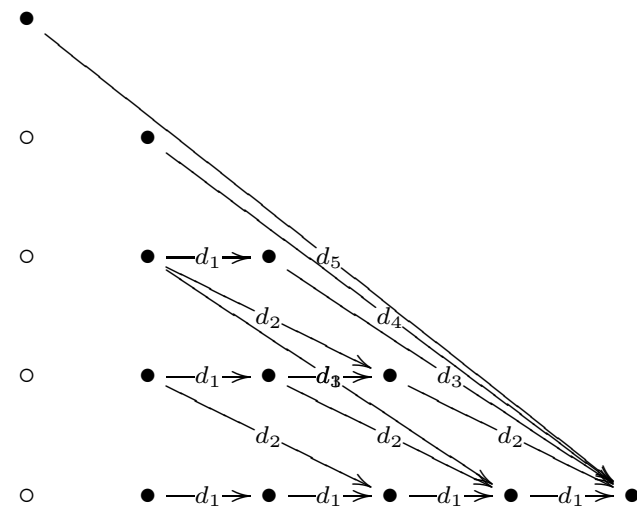

Figure 1. Possible nonzero differentials when $n=4$

Using $\rho=\alpha_{0}+\cdots+\alpha_{n}-\beta_{0}$, this becomes

$$
0 \rightarrow \underbrace{\mathcal{O}_{X}\left(-\beta_{0}\right)}_{\mathcal{F}^{0}} \rightarrow \underbrace{\bigoplus_{i} \mathcal{O}_{X}\left(\alpha_{i}-\beta_{0}\right)}_{\mathcal{F}^{1}} \rightarrow \cdots \rightarrow \underbrace{\bigoplus_{k} \mathcal{O}_{X}\left(\rho-\alpha_{k}\right)}_{\mathcal{F}^{n}} \rightarrow \underbrace{\mathcal{O}_{X}(\rho)}_{\mathcal{F}^{n+1}} \rightarrow 0
$$

We will now study the hypercohomology of the complex $\mathcal{F}^{\bullet}$.

Since (2.2) is exact, the hypercohomology $\mathbb{H}^{*}\left(X, \mathcal{F}^{\bullet}\right)$ vanishes identically. Hence we get a spectral sequence

$$
E_{1}^{p, q}=H^{q}\left(X, \mathcal{F}^{p}\right) \Rightarrow 0
$$

where the differential $d_{1}^{p, q}: E_{1}^{p, q} \rightarrow E_{1}^{p+1, q}$ is induced by the map $\mathcal{F}^{p} \rightarrow \mathcal{F}^{p+1}$ of the Koszul complex (2.2).

We compute $E_{1}^{p, q}$ as follows. When $p=0$,

$$
\operatorname{dim}\left(E_{1}^{0, q}\right)=\operatorname{dim}\left(H^{q}\left(X, \mathcal{O}_{X}\left(-\beta_{0}\right)\right)\right)= \begin{cases}0, & q<n, \\ 1, & q=n,\end{cases}
$$

where $q=n$ uses the isomorphism $H^{n}\left(X, \mathcal{O}_{X}\left(-\beta_{0}\right)\right)=H^{n}\left(X, \mathcal{O}_{X}(K)\right) \simeq \mathbb{C}$ given by the trace map.

When $p>0$, we have

$$
\mathcal{F}^{p}=\bigoplus_{|J|=p} \mathcal{O}_{X}\left(\alpha_{J}-\beta_{0}\right), \quad \alpha_{J}=\sum_{j \in J} \alpha_{j} .
$$

Then Corollary [1.3 and (2.1) imply that

$$
\operatorname{dim}\left(E_{1}^{p, q}\right)=\sum_{|J|=p} \operatorname{dim}\left(H^{q}\left(X, \mathcal{O}_{X}\left(\alpha_{J}-\beta_{0}\right)\right)\right)=\sum_{|J|=p, \operatorname{dim}\left(\Delta_{J}\right)=n-q} l^{*}\left(\Delta_{J}\right) .
$$

Our assumption that $\left\{\Delta_{i}, 0 \leq i \leq n\right\}$ is essential implies that $\operatorname{dim}\left(\Delta_{J}\right) \geq|J|$ when $|J| \leq n$. Combining this with $|J|=p, \operatorname{dim}\left(\Delta_{J}\right)=n-q$ from (2.4), we see that $n-q \geq p$ when $p \leq n$ in the last summation of (2.4). Thus

$$
E_{1}^{p, q}=0 \text { when } p+q>n \text {, except for } E_{1}^{n+1,0}=S_{\rho} .
$$

When $n=4,(2.3)$ and (2.5) give the picture shown in Figure 1 of all possible nonzero differentials in the spectral sequence. 
Now consider $E_{r}^{n+1,0}$. By (2.2), $E_{1}^{n, 0} \rightarrow E_{1}^{n+1,0} \rightarrow E_{1}^{n+2,0}$ is

$$
\bigoplus_{k} S_{\rho-\alpha_{k}} \longrightarrow S_{\rho} \longrightarrow 0
$$

Since this comes from the Koszul complex of the $f_{i}$, the image of the first map is $I_{\rho}$. It follows that

$$
E_{2}^{n+1,0}=(S / I)_{\rho} .
$$

All differentials starting from $E_{2}^{n+1,0}$ obviously vanish, and the only differentials which can map to this position are those on the "diagonal" $p+q=n$ as in Figure 1. Furthermore, on the diagonal, the only nonzero differentials $d_{r}^{n-q, q}$ are $d_{q+1}^{n-q, q}$ : $E_{q+1}^{n-q, q} \rightarrow E_{q+1}^{n+1,0}$. Since the spectral sequence converges to zero, it follows easily that

$$
\operatorname{dim}\left((S / I)_{\rho}\right) \leq \sum_{q=1}^{n} \operatorname{dim}\left(E_{1}^{n-q, q}\right) .
$$

By (2.3) and (2.4), we get the upper bound of part (1) of the theorem. As for the lower bound, note that the differentials

$$
d_{n+1}^{0, n}: E_{n+1}^{0, n} \rightarrow E_{q+1}^{n+1,0} \quad \text { and } \quad d_{n}^{1, n-1}: E_{n}^{1, n-1} \rightarrow E_{q+1}^{n+1,0}
$$

must be injective since the spectral sequence converges to 0 and nothing can map to these positions. (In Figure 1, these correspond to the differentials $d_{5}$ and $d_{4}$.) Using (2.3) and (2.4) again, we get the lower bound of part (1).

Turning to part (2) of the theorem, suppose that every differential $d_{r}$ mapping to $E_{r}^{p, q}$ is zero for $p+q=n$ and $q>0$. Since the spectral sequence converges to zero, this implies that

$$
\operatorname{dim}\left((S / I)_{\rho}\right)=\sum_{q=1}^{n} \operatorname{dim}\left(E_{1}^{n-q, q}\right) .
$$

From this, we easily get the desired formula for the codimension. Hence it suffices to prove that these differentials vanish when condition (a), (b) or (c) is satisfied by any $J$ with $1 \leq|J| \leq n-2$. A differential mapping to the diagonal $p+q=n$ originates from the "sub-diagonal" $p+q=n-1$. Thus we need to prove that all differentials

$$
d_{r}^{p, q}: E_{r}^{p, q} \longrightarrow E_{r}^{p+r, q-r+1}
$$

vanish when $p+q=n-1$ and $q-r+1>0$ (i.e., $r \leq q$ ).

To analyze this, first note that for arbitrary $(p, q)$, Corollary 1.3 implies that

$$
E_{1}^{p, q}=\bigoplus_{|J|=p, \operatorname{dim}\left(\Delta_{J}\right)=n-q} H_{J}
$$

where

$$
H_{J}=H^{q}\left(X, \mathcal{O}_{X}\left(\alpha_{J}-\beta_{0}\right)\right)=\bigoplus_{m \in M \cap \operatorname{int}\left(\Delta_{J}\right)} \mathbb{C} \cdot \chi^{m} .
$$

In particular, when $p+q=n-1$, the description of $E_{1}^{p, q}$ becomes

$$
E_{1}^{p, q}=\bigoplus_{\operatorname{dim}\left(\Delta_{J}\right)=|J|+1=n-q} H_{J}
$$


The subsets $J \subset\{0, \ldots, n\}$ in this direct sum satisfy

$$
|J|=p=n-q-1 \leq n-2 \quad \text { and } \quad \operatorname{dim}\left(\Delta_{J}\right)=n-q=|J|+1 .
$$

Every $J$ in the discussion which follows will satisfy (2.8).

Now consider $d_{1}^{p, q}: E_{1}^{p, q} \rightarrow E_{1}^{p+1, q}$ for $p+q=n-1$ and $q \geq 1$. By (2.7) and (2.8), we see that $H_{J}=0$ when $J$ satisfies conditions (a) or (b) of part (2) of the theorem. Now suppose $J$ satisfies condition (c). Since $d_{1}$ comes from the Koszul complex, (2.6) for $(p+1, q)$ shows that $d_{1}^{p, q}$ restricted to $H_{J}$ is a map

$$
d_{1}^{p, q}: H_{J} \longrightarrow \bigoplus_{i \notin J, \operatorname{dim}\left(\Delta_{J \cup\{i\}}\right)=n-q} H_{J \cup\{i\}}
$$

By condition (c), we know that $\operatorname{dim}\left(\Delta_{J \cup\{i\}}\right)>|J|+1$ for $i \notin J$. But $|J|=n-q-1$ by (2.8), so that $\operatorname{dim}\left(\Delta_{J \cup\{i\}}\right)>n-q$. Comparing this to the above description of $d_{1}^{p, q}$ shows that $d_{1}^{p, q}=0$ when $p+q=n-1$ and $q \geq 1$, as claimed.

Next consider $d_{2}^{p, q}: E_{2}^{p, q} \rightarrow E_{2}^{p+2, q-1}$, where $p+q=n-1$ and $q \geq 2$. To understand this map, we will recall its definition which follows from the Snake Lemma. Let $\mathcal{U}$ be a Leray covering of $X$, so that the cohomology groups $H^{q}\left(X, \mathcal{F}^{p}\right)$ can be identified with the Cech cohomology of $X$ with respect to this covering. As usual, we call $\delta$ the maps between cochain groups. Also let

$$
Z^{q}\left(\mathcal{U}, \mathcal{F}^{p}\right) \subset C^{q}\left(\mathcal{U}, \mathcal{F}^{p}\right)
$$

denote the corresponding Cech group and its subgroup of cocycles. Then we compute $d_{2}$ using the following diagram:

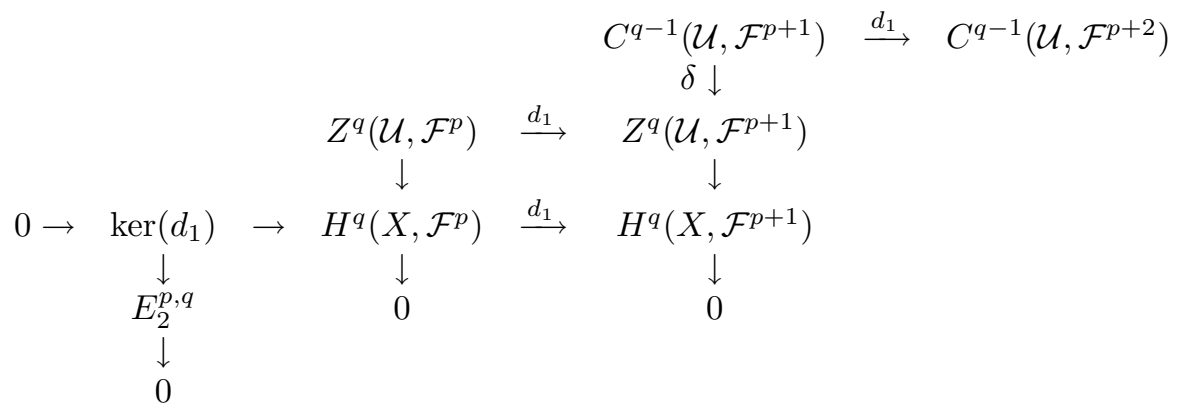

In this diagram, $s \in E_{2}^{p, q}$ lifts to an element of $E_{1}^{p, q}=H^{q}\left(X, \mathcal{F}^{p}\right)$ represented by $s^{\prime} \in Z^{q}\left(\mathcal{U}, \mathcal{F}^{p}\right)$. Then

$$
d_{1}\left(s^{\prime}\right) \in Z^{q}\left(\mathcal{U}, \mathcal{F}^{p+1}\right)
$$

represents $0 \in H^{q}\left(X, \mathcal{F}^{p+1}\right)$. Thus $d_{1}\left(s^{\prime}\right)$ lifts to $Z^{q-1}\left(\mathcal{U}, \mathcal{F}^{p+1}\right)$, i.e., $d_{1}\left(s^{\prime}\right)=\delta\left(s^{\prime \prime}\right)$ for some $s^{\prime \prime} \in Z^{q-1}\left(\mathcal{U}, \mathcal{F}^{p+1}\right)$. It is then easy to see that

$$
d_{1}\left(s^{\prime \prime}\right) \in Z^{q-1}\left(\mathcal{U}, \mathcal{F}^{p+2}\right),
$$

so that $d_{1}\left(s^{\prime \prime}\right)$ represents an element of $E_{1}^{p+2, q-1}=H^{q-1}\left(X, \mathcal{F}^{p+2}\right)$. This is killed by $d_{1}$ and represents $d_{2}^{p, q}(s) \in E_{2}^{p+2, q-1}$. It follows that $d_{2}$ is constructed by applying $d_{1}$ twice to suitable liftings.

Since $d_{1}^{p, q}$ vanishes, $E_{2}^{p, q}$ is a quotient of $E_{1}^{p, q}$. So we may assume that $s \in E_{2}^{p, q}$ comes from $H_{J}$ for some $J$ satisfying (2.8). As before, this vanishes if $J$ satisfies conditions (a) or (b) of part (2). Now assume $J$ satisfies condition (c). Then as above $s$ gives $s^{\prime} \in Z^{q}\left(\mathcal{U}, \mathcal{F}^{p}\right)$ where we only use the summand of $\mathcal{F}^{p}$ corresponding to $J$. Since $d_{1}$ comes from the Kozsul complex, we see from (2.9) that $d_{1}\left(s^{\prime}\right)$ only 
involves summands of $\mathcal{F}^{p+1}$ corresponding to $J \cup\{i\}$ for $i \notin J$. Then the same is true for $s^{\prime \prime}$, so that in (2.10),$d_{1}\left(s^{\prime \prime}\right)$ only involves summands of $\mathcal{F}^{p+1}$ corresponding to $J \cup\{i, j\}$ for $i, j \notin J$ and $i \neq j$.

This shows that $d_{2}^{p, q}(s) \in E_{2}^{p+2, q-1}$ is represented by an element of $E_{1}^{p+2, q-1}$ involving only summands $H_{J \cup\{i, j\}}$ for $i, j \notin J$ and $i \neq j$. Yet to actually appear in $E_{1}^{p+2, q-1}$, the summand must satisfy $n-(q-1)=\operatorname{dim}\left(\Delta_{J \cup\{i, j\}}\right)$ by (2.6). This is impossible since condition (c) implies that

$$
\operatorname{dim}\left(\Delta_{J \cup\{i, j\}}\right)>|J|+2=(n-q-1)+2=n-(q-1) .
$$

It follows that $d_{2}^{p, q}=0$ when $p+q=n-1$ and $q \geq 2$.

The argument for general $r$ is similar. Here, $p+q=n-1, q \geq r$, and $d_{1}^{p, q}, \ldots, d_{r-1}^{p, q}$ vanish. Then $E_{r}^{p, q}$ is a quotient of $E_{1}^{p, q}$, so we may assume that $s \in E_{r}^{p, q}$ comes from $H_{J}$ for $J$ as in (2.8). Since $d_{1}$ comes from the Koszul complex and $d_{r}$ is obtained by applying $d_{1} r$ times to suitable liftings, we see that $d_{r}^{p, q}(s) \in E_{r}^{p+r, q-r+1}$ is represented by an element of $E_{1}^{p+r, q-r+1}$ involving only summands $H_{J \cup I}$ for $|I|=r$ and $I \cap J=\emptyset$. In order to appear in $E_{1}^{p+r, q-r+1}$, the summand must satisfy $n-(q-r+1)=\operatorname{dim}\left(\Delta_{J \cup I}\right)$ by (2.6). This is impossible since condition (c) implies that

$$
\operatorname{dim}\left(\Delta_{J \cup I}\right)>|J|+|I|=(n-q-1)+r=n-(q-r+1) .
$$

It follows that $d_{r}^{p, q}=0$ when $p+q=n-1$ and $q \geq r$. This completes the proof of the theorem.

Theorem 2.2 has the following corollaries.

Corollary 2.3. Let $\Delta_{0}, \ldots, \Delta_{n}$ be an essential family of lattice polytopes in $\mathbb{R}^{n}$. Then the Minkowski sum $\Delta_{0}+\cdots+\Delta_{n}$ has at least one interior lattice point.

Proof. If $X_{\Delta}$ is the toric variety determined by $\Delta=\Delta_{0}+\cdots+\Delta_{n}$, then $\operatorname{dim}\left(S_{\rho}\right)$ is the number of interior lattice points of $\Delta$. The lower bound given by Theorem 2.2 implies that $\operatorname{dim}\left(S_{\rho}\right) \geq \operatorname{dim}\left((S / I)_{\rho}\right) \geq 1$, and the corollary follows.

Remark 2.4. Corollary 2.3 may fail if $\Delta_{0}, \ldots, \Delta_{n}$ are not essential. For example, suppose that $\Delta_{1}+\cdots+\Delta_{n}$ has dimension $n-1$ and $\Delta_{0}$ is an interval of length one relative to an integral linear functional constant on the affine hyperplane containing $\Delta_{1}+\cdots+\Delta_{n}$. It is easy to see that $\Delta_{0}+\cdots+\Delta_{n}$ has no interior lattice points. We are grateful to Günter Ziegler for this observation.

Corollary 2.5. Suppose that $X$ is a complete toric variety of dimension $n$. Let $\alpha_{i} \in \operatorname{Pic}(X), 0 \leq i \leq n$, be globally generated and assume that $f_{i} \in S_{\alpha_{i}}, 0 \leq i \leq n$, do not vanish simultaneously on $X$. Then:

(1) If the polytopes $\Delta_{i}$ all have dimension $n$, then

$$
\operatorname{dim}\left((S / I)_{\rho}\right)=1 .
$$

(2) If $n=2$ and $\left\{\Delta_{0}, \Delta_{1}, \Delta_{2}\right\}$ is essential, then

$$
\operatorname{dim}\left((S / I)_{\rho}\right)=1+\sum_{\operatorname{dim}\left(\Delta_{i}\right)=1} l^{*}\left(\Delta_{i}\right)
$$

Proof. This follows immediately from part (2) of Theorem 2.2. 
Remark 2.6. In part (1) of Corollary 2.5, the hypothesis that $\alpha_{i}$ is globally generated and $\Delta_{i}$ has dimension $n$ is equivalent to assuming that $\alpha_{i}$ is the class of a big and nef divisor on $X$.

When $n=3$, the conditions of part (2) of Theorem 2.2 are equivalent to the assumption that if $\operatorname{dim}\left(\Delta_{i}\right)=2$, then either $\Delta_{i}$ has no interior lattice points or $\operatorname{dim}\left(\Delta_{i}+\Delta_{j}\right)=3$ for all $j \neq i$. It follows that Theorem[2.2 computes the codimension in the critical degree for many but not all cases of essential supports when $X$ has dimension 3.

There is one case where further general results are possible.

Theorem 2.7. Suppose that $X$ is a complete toric variety of dimension $n \geq 3$. Let $\alpha_{i} \in \operatorname{Pic}(X), 0 \leq i \leq n$, be globally generated and assume that the corresponding family of polytopes $\left\{\Delta_{i}, 0 \leq i \leq n\right\}$ is essential and satisfies

$$
\operatorname{dim}\left(\Delta_{i}\right) \in\{1, n-1, n\}, \quad i=0, \ldots, n .
$$

Let $f_{i} \in S_{\alpha_{i}}, 0 \leq i \leq n$, and assume the following two conditions:

(1) The $f_{i}$ do not vanish simultaneously on $X$.

(2) For every $J \subset\{0, \ldots, n\}$ with $\operatorname{dim}\left(\Delta_{J}\right)=|J|=n-1$ and $\operatorname{dim}\left(\Delta_{i}\right)=n-1$ for at least one $i \in J$, the equations on $X_{\Delta_{J}}$ given by $f_{j}=0$ for $j \in J$ have only finitely many solutions, all of which lie in the torus of $X_{\Delta_{J}}$.

Then the codimension of $I_{\rho}$ in $S_{\rho}$ is given by

$$
\operatorname{dim}\left((S / I)_{\rho}\right)=1+\sum_{\operatorname{dim}(\Delta J)=|J|<n}\left(\sum_{\mathcal{J} \subset J}(-1)^{|J|-|\mathcal{J}|} l_{|J|}^{*}\left(\sum_{\ell \in \mathcal{J}} P_{\ell}\right)\right),
$$

where, for a lattice polytope $\Delta$ and integer $k \geq 0$,

$$
l_{k}^{*}(\Delta)= \begin{cases}0, & \operatorname{dim}(\Delta) \neq k, \\ l^{*}(\Delta), & \operatorname{dim}(\Delta)=k .\end{cases}
$$

The condition (2.11) is still rather restrictive, yet examples with $n=4$ show that the formula (2.12) can fail when we omit (2.11). Furthermore, when we do assume (2.11), condition (2) on the $f_{i}$ is probably unnecessary, yet we cannot figure out how to prove the theorem without using this hypothesis. For these reasons, we omit the proof of Theorem 2.7 .

\section{REFERENCES}

[1] V. Batyrev, Variations of the mixed Hodge structure of affine hypersurfaces in algebraic tori, Duke Math. J. 69 (1993), 349-409. MR1203231 (94m:14067)

[2] V. Batyrev and L. Borisov, On Calabi-Yau complete intersections in toric varieties, in Higherdimensional complex varieties (Trento, 1994), de Gruyter, Berlin, 1996, 39-65. MR1463173 (98j:14052)

[3] V. Batyrev and E. Materov, Toric residues and mirror symmetry, Mosc. Math. J. 2 (2002), 435-475. MR1988969 (2005a:14070)

[4] E. Cattani, D. Cox, and A. Dickenstein, Residues in toric varieties, Compositio Math. 108 (1997), 35-76. MR1458757 (98f:14039)

[5] E. Cattani and A. Dickenstein, A global view of residues in the torus, J. Pure Appl. Algebra 117/118 (1997), 119-144. MR 1457836 (98i:14050)

[6] E. Cattani, A. Dickenstein, and B. Sturmfels, Rational hypergeometric functions, Compositio Mathematica 128 (2001), 217-240. MF 1850183 (2003f:33016)

[7] D. Cox, Toric residues, Ark. Mat. 34 (1996), 73-96. MR1396624 (97e:14062)

[8] C. D'Andrea and A. Khetan, Macaulay style formulas for toric residues, preprint, 2003, math.AG/0307154. 
[9] A. Mavlyutov, On the chiral ring of Calabi-Yau hypersurfaces in toric varieties, Compositio Math. 138 (2003), 289-336. MR2019444 (2004m:14085)

[10] M. Mustaţă, Vanishing theorems on toric varieties, Tohoku Math. J. 54 (2002), 451-470. MR 1916637 (2003e:14013)

[11] B. Sturmfels, On the Newton polytope of the resultant, J. Algebraic Combin. 3 (1994), 207236. MR $1268576 \mid(95 \mathrm{j}: 52024)$

Department of Mathematics and Computer Science, Amherst College, Amherst, MasSACHUSETTS 01002-5000

E-mail address: dac@cs.amherst.edu

Departamento de Matemática, F.C.E. y N., Universidad de Buenos Aires, Cuidad Universitaria-Pabellón I, 1428 Buenos Aires, Argentina

E-mail address: alidick@dm.uba.ar 